\title{
Mid-infrared electroluminescence at room temperature from InAsSb multi- quantum-well light-emitting diodes
}

\author{
A. Krier, ${ }^{\text {a) }}$ M. Stone, and Q. D. Zhuang \\ Department of Physics, Lancaster University, Lancaster LA1 4YB, United Kingdom \\ Po-Wei Liu, G. Tsai, and H. H. Lin \\ Department of Electrical Engineering, National Taiwan University, Taipei 106, Taiwan and Institute of \\ Electronic Engineering, National Taiwan University, Taipei 106, Taiwan
}

(Received 24 May 2006; accepted 6 July 2006; published online 30 August 2006)

\begin{abstract}
Room-temperature electroluminescence is reported from InAsSb multiple-quantum-well light-emitting diodes. The diodes exhibited emission in the mid-infrared peaking near $4 \mu \mathrm{m}$. The spectral dependence on injection current at $4 \mathrm{~K}$ was investigated and two transitions were identified, centered at 4.05 and $3.50 \mu \mathrm{m}$, which are associated with the eigenstates of the confined holes inside the quantum well. The use of an Sb predeposition and As flux surface exposure during epitaxial growth was observed to have a major effect on the electroluminescence output. (C) 2006 American Institute of Physics. [DOI: 10.1063/1.2339036]
\end{abstract}

The development of light sources and detectors for the mid-infrared $(2-5 \mu \mathrm{m})$ spectral region continues to be of much academic and technological interest. If suitable optoelectronic components could be made available, one can envisage a wide variety of applications, including chemical process control, environmental monitoring of atmospheric pollution, noninvasive medical diagnosis, tunable IR spectroscopy, and free space optical communications. In $\mathrm{As}_{1-x} \mathrm{Sb}_{x}$ alloys are promising materials for this spectral region; however, strong nonradiative Auger recombination in these materials results in low room-temperature quantum efficiencies in practical devices. One possible solution is to fabricate type II multiple-quantum-well (MQW) structures and make use of the two dimensional confinement to suppress Auger recombination and improve electroluminescence output.

Previous work in our laboratory using liquid phase epitaxial growth of InAsSb bulk alloys has resulted in lightemitting diodes (LEDs) which operate at $300 \mathrm{~K}$ with emission peaking near $4.2 \mu \mathrm{m}$ appropriate for $\mathrm{CO}_{2}$ detection. ${ }^{1} \mathrm{In}$ recent years MQW structures containing InAsSb have been used in the active region of diode lasers grown on $\mathrm{GaSb}$ and GaAs substrates by molecular beam epitaxy (MBE) or metalorganic vapor phase epitaxy (MOVPE)., ${ }^{2,3}$ By comparison, there have been very few reports of LEDs based on InAsSb MQW exhibiting emission within the $2-5 \mu \mathrm{m}$ spectral range at room temperature. ${ }^{4}$ Previously, we have reported on the photoluminescence (PL) from InAs ${ }_{1-x} \mathrm{Sb}_{x}$ quantum dot in the mid-infrared near $4 \mu \mathrm{m} .{ }^{5}$ In the present work we report on the observation of electroluminescence (EL) from $\mathrm{InAs}_{0.87} \mathrm{Sb}_{0.13} / \mathrm{InAs} \mathrm{MQW}$ light-emitting diodes grown by MBE.

The InAsSb/InAs MQW samples for this work were grown on (100) $p^{+}$-InAs substrates using a VG-V80H MBE system. Two cracker cells were used to provide the $\mathrm{Sb}$ and $\mathrm{As}_{2}$ beams. A thermal effusion $\mathrm{K}$ cell was used to provide the In flux. A $p^{+}$-InAs buffer layer with a thickness of $300 \mathrm{~nm}$ was grown at $450{ }^{\circ} \mathrm{C}$, followed by a ten-period InAsSb/InAs MQW with $13 \% \mathrm{Sb}$ composition which was grown at $466{ }^{\circ} \mathrm{C}$. The thicknesses of the InAsSb well and

\footnotetext{
${ }^{a)}$ Electronic mail: a.krier@lancaster.ac.uk
}

InAs barrier were 7 and $24 \mathrm{~nm}$, respectively. Finally, a $n^{+}$ layer was grown to complete the simple homojunction $p-i-n$ diode structure. In situ reflection high energy electron diffraction was used to monitor surface reconstruction. After growth the samples were characterized using double crystal x-ray diffraction and PL or EL spectroscopy to study their structural and optical properties. For EL measurements the as-grown sample was processed into $1 \mathrm{~mm}$ diameter mesaetched LEDs using standard photolithographic techniques. The resulting diodes were mounted onto TO-46 headers for testing and a quasicontinuous current (50\% duty cycle) at $1 \mathrm{kHz}$ was used to excite EL in the LEDs. The corresponding low temperature luminescence spectra were measured using a Bentham $0.3 \mathrm{~m}$ monochromator and a $77 \mathrm{~K}$ InSb photodiode to detect the radiation. We investigated the influence of the As and $\mathrm{Sb}$ switching sequences employed during MBE growth on the structural properties and also on the resulting EL emission properties.

Figure 1 shows the high resolution $x$-ray diffraction

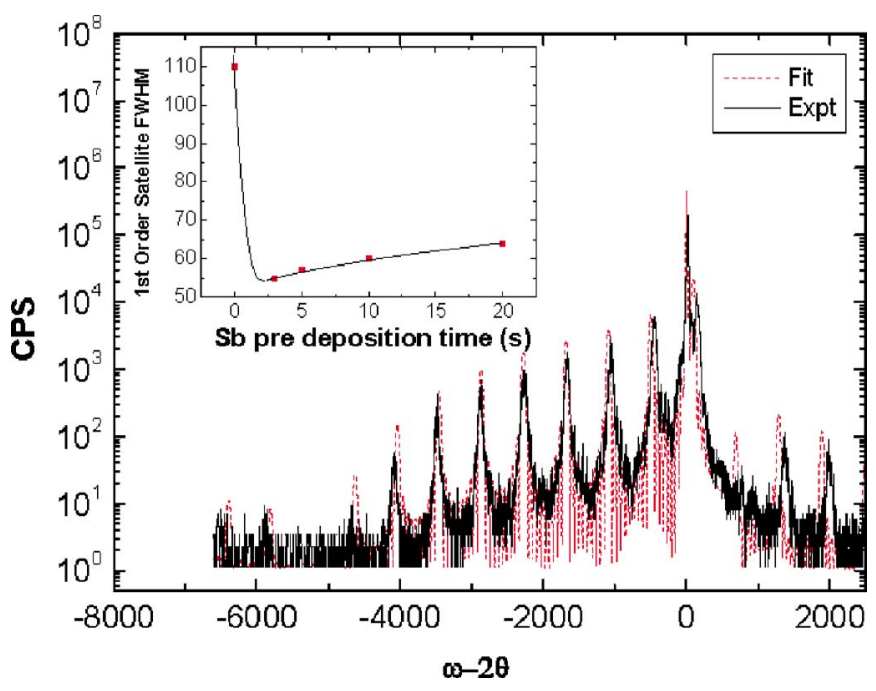

FIG. 1. (Color online) High resolution x-ray diffraction spectra obtained from one of the InAsSb/InAs MQW samples; solid line-experimental results, dashed line - simulated data. Inset shows FWHM dependence on $\mathrm{Sb}$ predeposition time. 


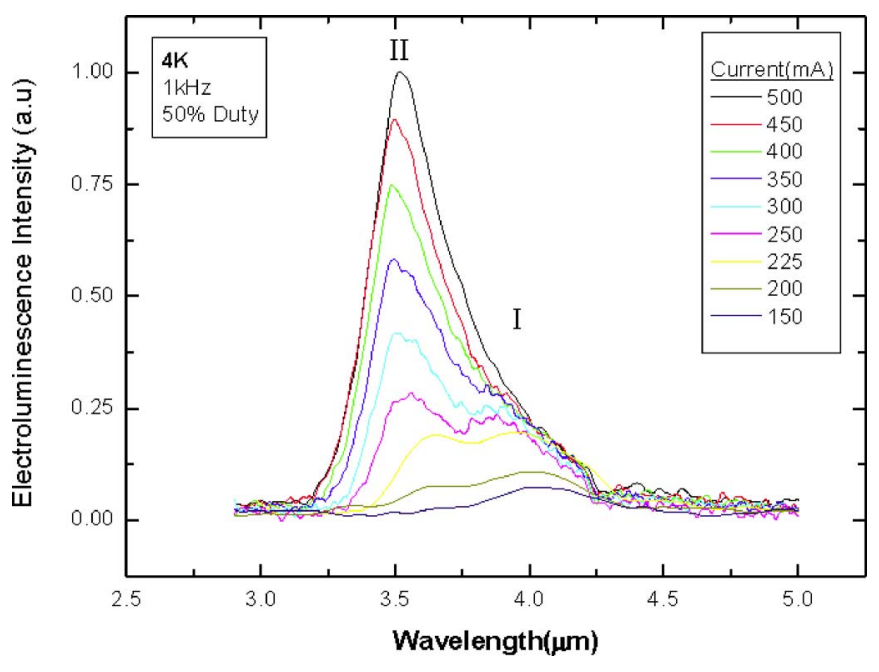

FIG. 2. (Color online) $4 \mathrm{~K}$ electroluminescence emission spectra observed from the InAsSb MQW LED using different injection currents.

spectrum measured from a sample containing ten InAsSb quantum wells. A number of well defined satellite peaks are easily observed and the full width at half maximum (FWHM) of these peaks is a good indicator of the quality of the MQW structure. To obtain the narrowest satellite peaks, it was necessary to carefully control the As and $\mathrm{Sb}$ flux switching sequence. We found that the best results were obtained when a minimum of $3 \mathrm{~s}$ Sb predeposition was used prior to the growth of the InAsSb QW, followed by a $20 \mathrm{~s}$ As deposition before growth of the InAs barrier. In this way we obtained the most abrupt interfaces and the brightest EL emission.

Figure 2 shows the $4 \mathrm{~K}$ EL spectrum obtained from one of the InAsSb/InAs MQW LEDs using 150-500 mA quasi-cw injection current. At low injection only one peak (I) is observed which exhibits a small blueshift. Above about $250 \mathrm{~mA}$ this peak saturates and a second higher energy peak (II) is observed. The spectra may be interpreted based on the transitions originating as shown in Fig. 3, where the band lineup is depicted as type II with strong hole confinement. ${ }^{6}$ Peaks I $(4.05 \mu \mathrm{m})$ and II $(3.50 \mu \mathrm{m})$ can be assigned to recombination from the InAs conduction band to the heavy hole and light hole confined states in the InAsSb quantum well. These transitions are in excellent agreement with calculated values. We also noted that no emission $(\sim 3.0 \mu \mathrm{m})$ from the InAs barriers or from the InAs substrate was observed, which suggests strong carrier localization at the QW.

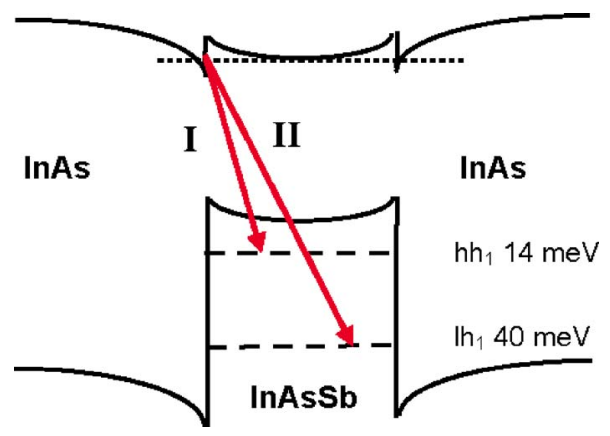

FIG. 3. (Color online) Schematic diagram showing the type II $\mathrm{InAs}_{1-x} \mathrm{Sb}_{x} / \mathrm{InAs}$ quantum well and the transitions corresponding to peaks I

and II observed in the $4 \mathrm{~K} \mathrm{EL}$ spectra.
Downloaded 11 Feb 2009 to 140.112 .113 .225 . Redistribution subject to AlP license or copyright; see http://apl.aip.org/apl/copyright.jsp
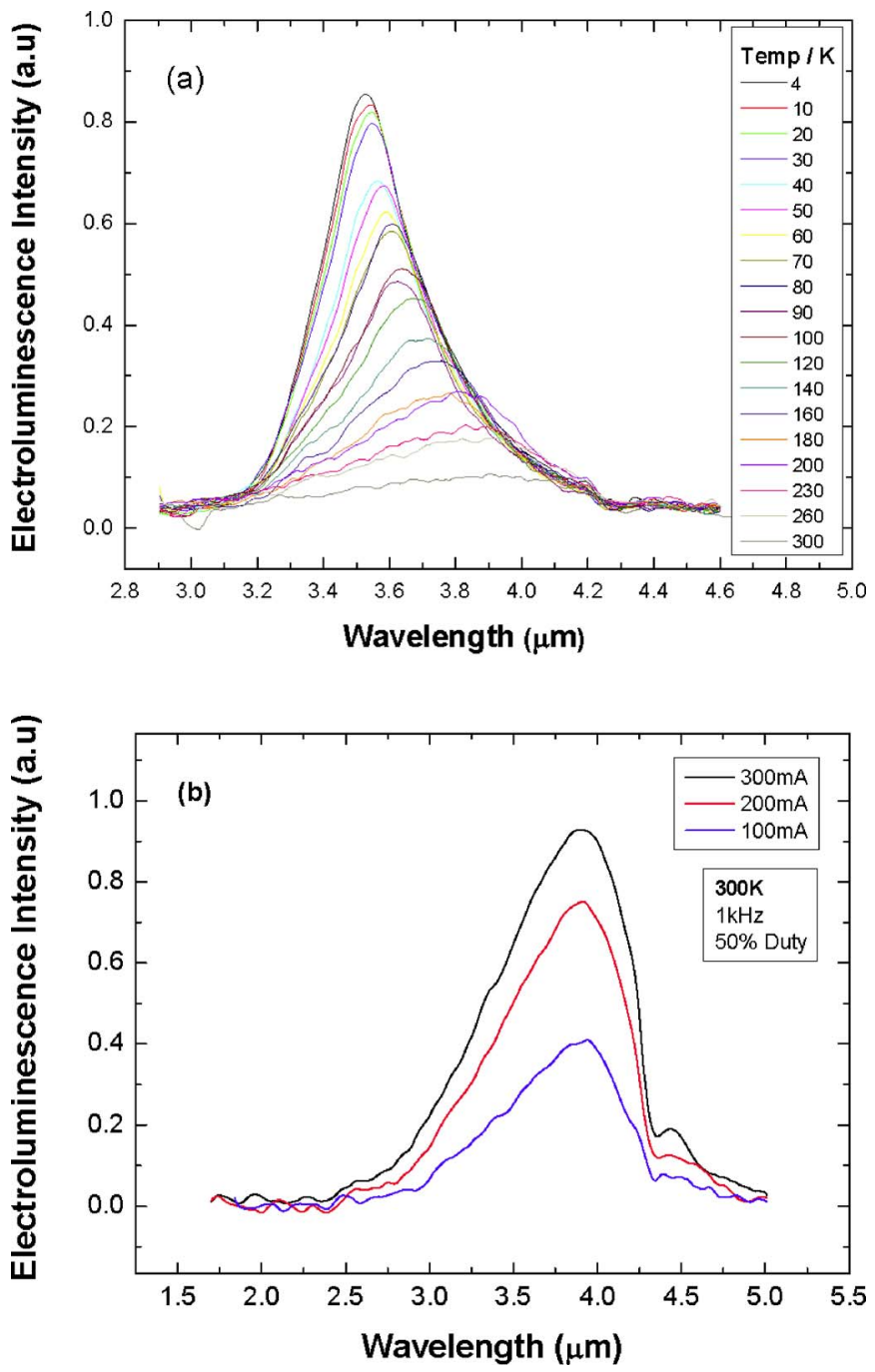

FIG. 4. (Color online) EL spectrum of the MQW LED (a) at various temperatures using an injection current of $500 \mathrm{~mA}$. (b) The dependence of the room-temperature emission spectrum on current.

Figure 4(a) shows the electroluminescence emission spectra obtained from the InAsSb MQW LED measured with increasing temperature over the range of 4-300 K using $500 \mathrm{~mA}$ injection current. The dominant emission peak (II) decreased steadily with increasing temperature. On approaching room temperature, the emission broadened and a high energy tail developed, presumably associated with some recombination originating within the InAs barriers as carriers become released from the MQW. The peak intensity quenched by only a factor of 7 as a result of the carrier confinement compared with a value of $>15$ typically observed in the bulk alloy. The redshift of the EL peak emission corresponds to the usual band gap narrowing with temperature. Figure 4(b) shows the EL emission observed at room temperature for three different injection currents using quasi-cw operation (50\% duty cycle is the preferred mode of operation for many perceived practical applications). The emission peaks near $4 \mu \mathrm{m}$ and moves slightly to longer wavelength as the current is increased due to Joule heating in the diode. The asymmetry in the spectra on the long wavelength side is partly due to absorption from $\mathrm{CO}_{2}$ in the laboratory atmosphere.

The LED output power under quasicontinuous drive conditions ( $1 \mathrm{kHz}$ at $50 \%$ duty cycle) was measured at room 
temperature using an integrating sphere and calibrated $\mathrm{PbSe}$ photodetector. The corresponding internal quantum efficiency was estimated to be $0.2 \%$. This resulted in an output power of $1.4 \mu \mathrm{W}$ at room temperature. These values can be improved since the LED is a homojunction diode and the present devices have not yet been optimized with respect to the active region thickness or the $p^{+}$and $n^{+}$doping concentrations.

The observed improvement in the InAsSb MQW as a result of predeposition of $\mathrm{Sb}$ is in good agreement with previous results obtained using MOVPE. Pillai et al. have studied the effect of $\mathrm{Sb}$ predeposition on the compositional profile of $\operatorname{InAs}_{0.80} \mathrm{Sb}_{0.20} /$ InAs superlattices and observed $\mathrm{Sb}$ segregation for samples grown with no predeposition. ${ }^{7}$ For samples grown with $0.8 \mathrm{ML}$ Sb predeposition, sharper InAsSb on InAs interfaces were obtained. Sb coverages of $>1$ $\mathrm{ML}$ resulted in broader $\mathrm{x}$-ray satellite peaks and rougher interfaces. Kaspi and Evans showed that an Sb floating layer forms on a III-V alloy surface. ${ }^{8}$ By using the fact that $\mathrm{Sb}$ is more volatile than As and choosing a suitable As exposure time, the Sb floating layer can be removed. In our work a $20 \mathrm{~s}$ As exposure was used to remove this floating layer and thus prevent $\mathrm{Sb}$ incorporation into the InAs barrier layers. Our results indicate that these benefits carry over to the luminescence efficiency with the best EL being obtained from the samples with the sharpest interfaces. We also found that the luminescence emission intensity from the MQW is enhanced compared with samples grown without any predeposition or As exposure. This enhancement becomes more significant as the $\mathrm{Sb}$ content in the MQW increases. A comparison of the emission spectra from samples prepared with and without the $\mathrm{Sb}$ predeposition is shown in Fig. 5. The MQW grown with predeposition exhibit emission at shorter wavelengths which we associate with reduced bowing in the valence band arising from the more abrupt interfaces with less structural disorder. We note that the bowing parameter in InAsSb is almost entirely determined by structural disorder. ${ }^{9}$ Hence, changes in structural perfection will have a significant effect on the bowing parameter and consequently on the dependence of band gap on composition and the corresponding transition energy of the InAsSb in the MQW. Previously we reported the InAsSb conduction band bowing to be near $40 \%$ for samples grown without $\mathrm{Sb}$ predeposition. ${ }^{10}$ The present results indicate that a value near $60 \%$ would be more appropriate for MQW grown using $\mathrm{Sb}$ predeposition.

In summary, mid-infrared electroluminescence from $\operatorname{InAs}_{0.87} \mathrm{Sb}_{0.13} /$ InAs MQW homojunction LEDs grown by MBE has been observed near $4.0 \mu \mathrm{m}$. The recombination was identified as originating from type II transitions involv-

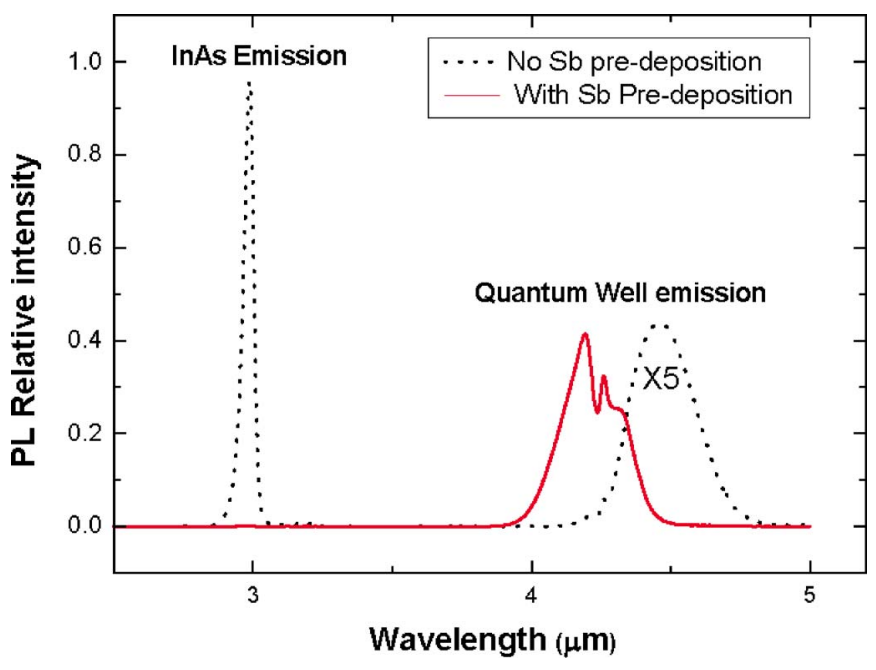

FIG. 5. (Color online) Comparison of the PL emission spectra from samples prepared with and without the Sb predeposition and As exposure.

ing confined holes and persisted up to room temperature with improved quenching behavior compared with the equivalent bulk alloy. The use of $\mathrm{Sb}$ predeposition during epitaxial growth produced MQWs with more abrupt interfaces which had a major effect on the electroluminescence. Both the best luminescence and the narrowest $\mathrm{x}$-ray satellite peak width were obtained for an $\mathrm{Sb}$ predeposition time of $3 \mathrm{~s}$. With some optimization of the structure and with the addition of an electron blocking barrier to confine the injected electrons, it should be possible to obtain significant further improvements in LED output and efficiency.

The authors wish to thank Kidde Ltd. for providing a CASE studentship for one of the authors (M.S.). The authors also wish to thank D. Campbell and G. Crook for valuable technical assistance and A. Godenir for X-ray measurements.

${ }^{1}$ A. Krier, H. H. Gao, V. V. Sherstnev, and Y. Yakovlev, J. Phys. D 32, 3117 (1999).

${ }^{2}$ E. R. Glaser, B. R. Bennett, B. V. Shanabrook, and R. Magno, Appl. Phys. Lett. 68, 3614 (1996).

${ }^{3}$ G. Park, O. B. Shchekin, D. L. Huffaker, and D. G. Deppe, Appl. Phys. Lett. 73, 3351 (1998).

${ }^{4}$ A. Stein, D. Puttjer, A. Behres, and K. Heime, IEE Proc.: Optoelectron. 145, 257 (1998).

${ }^{5}$ A. Krier, X. L. Huang, and A. Hammiche, Appl. Phys. Lett. 77, 3791 (2000).

${ }^{6}$ S. H. Wei and A. Zunger, Phys. Rev. B 52, 12039 (1995).

${ }^{7}$ M. Pillai, S. C. Theiring, S. A. Barnett, B. W. Wessels, A. Desikan, and E. P. Kvam, J. Cryst. Growth 208, 79 (2000).

${ }^{8}$ R. Kaspi and K. R. Evans, J. Cryst. Growth 175/176, 838 (1997).

${ }^{9}$ M. Ferhat, Phys. Status Solidi B 241, R38 (2004).

${ }^{10}$ P. W. Liu, G. Tsai, H. H. Lin, and A. Krier, OPT Conference (Tainan, Taiwan, 2005), paper. No. A-FR-II 4-2. 